\title{
Smart Solution for Agriculture Leaf Disease
}

\author{
Rajlaxmi Chavan, A. M. Rawate, A. S. Paymode
}

\begin{abstract}
Agriculture is a very broad term encompassing all aspects of crop production,Livestock farming, Fisheries, Forestry etc. It may be defined as the art, the science and the business of producing crops and livestock for man's use and employment. Agriculture is influenced by a large number of factors, some of which can be controlled by man(soil and irrigation) which others are beyond the control(climate). As we know Population pressure is increasing but area under cultivation is static, therefore more number of crops have to be grown on the same piece of land to increase the yield. As a result, intensive cropping has come into practice. for this purpose we are using the system which is pocket friendly as well as effective to the farmers. Through which they can get accurate information about their crop plants
\end{abstract}

Keywords: Opencv, Python, Numpy, Sensor, Raspberry Pi, GSM

\section{INTRODUCTION}

New technology has to be developed to overcome the effect of moisture stress under dryland conditions. As new varieties of crops with high yield potential become available package of practices have to be developed to exploit their full potential. Restoration of soil fertility, preparation of good seed bed, use of proper seed rates, correct dates of sowing for each improved variety, proper conservation and management of soil moisture and proper control of weeds are agronomic practices to make our limited land and water resources more productive.As the data from a 20 per cent sample is large enough to estimate crop area with a sufficient degree of precision at the all-India, State and district levels, crop area forecasts and final area estimates issued by the Ministry of Agriculture should be based on the results of the 20 per cent Timely Reporting Scheme(TRS) villages in the temporarily settled States and Establishment of an Agency for Reporting Scheme(EARAS) scheme villages in the permanently settled states. In the case of the North-Eastern States, Remote Sensing methodology should be used for this purpose after testing its viability.Farmers do all hard work effectively then also they doesn't get good price of their crops due to mediator. As per data, The ministry of Agriculture should prepare a well-documented manual of instructions on collection of wholesale prices of agricultural commodities. Latest tools of communication technology like e-mail should be availed of to ensure timely data flow of agricultural prices.

Revised Manuscript Received on August 27, 2020.

* Correspondence Author

Ms. Rajlaxmi P. Chavan*, MTech Student, Department of Electronics and Telecommunications Engineering, CSMSS, Chh. Shahu College of Engineering, Aurangabad (Maharashtra), India.

Prof. A.M.Rawate, Head of Department, Department of Electronics and Telecommunication Engineering, CSMSS, Chh. Shahu College of Engineering, Aurangabad (Maharashtra), India.

Prof. A.S. Paymode, Assistant Professor, Department of Electronics and Telecommunication Engineering, CSMSS, Chh. Shahu College of Engineering, Aurangabad (Maharashtra), India.

(C) The Authors. Published by Blue Eyes Intelligence Engineering and Sciences Publication (BEIESP). This is an open access article under the CC BY-NC-ND license (http://creativecommons.org/licenses/by-nc-nd/4.0/)
A system should be developed to secure a simultaneous data flow of agricultural prices from lower levels to the State as well as the Centre.For this problem, we want mono system which effectively do all needful things.

\section{PROBLEM IDENTIFICATION}

In olden days and today also, Farmers usually detect the crop disease by simply seeing to leaf of plant. Which makes them take hard theories on which fertilisers to use. It needs detailed knowledge the types of diseases and lot of experience required to make sure the actual disease detection. Some of diseases look almost same to farmers often leaves them puzzled. So in this scenario farmer makes wrong prediction of fertiliser. In case the farmer makes wrong choice or use more fertiliser than needed. It will destroyed whole plant production. Similar happened when we used excessive of water to the crop. Where moisture level of crops increased.To prevent this situation, we need correct and reliable information of fertilisers to use, to make the correct identification of diseases, and the ability to differentiate between two or more similar types of diseases in visuals. some work related to smart agriculture using IOT done successfully but in some cases it is expensive and vast. To overcome from this we can give all facility in one system with cost efficient way. In IOT based smart farming, a system is built for monitoring the crop field with the help of sensors and also farmers can monitor the field conditions from anywhere. It is highly efficient when compared with conventional approach.

\section{RELATED WORK}

Some already developed systems in the problem area which mentioned above:

Book Precision Agriculture [1] by TenryBrase, It provides an overview of precision farming concepts and the tools of precision farming system. An overview of latest technology which is essential for farming.

Designing Urban Agriculture[2] by April Philips, It represents strategies, tools and guidelines that enable informed decisions on planning, designing, budgeting, constructing, maintaining, marketing and increasing the sustainability of this re-invented city scrap.

IOT and Analytics for Agriculture[3] by Prasant Kumar Pattnaik, It represents recent holding on virtually every aspect of wireless IOT and Analytics for Agriculture. It gives brief account of IOT based monitoring systems for analysing the crop environment and methods for improving the efficiency of decision making based on analysis of harvest statistics. This book gives idea of technology we can implement in our work.

Published By:

Blue Eyes Intelligence Engineering

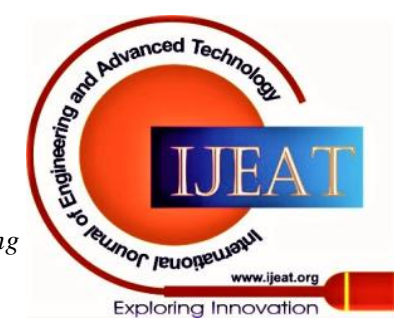




\section{Smart Solution for Agriculture Leaf Disease}

\section{SYSTEM DESIGN}

Software Used
A. OpenCv
B. SMTP Protocol
C. Python
D. Database

Hardware Used
A. Power Supply
B. Input Switch
C. Camera
D. Encloser
E. Indicators
F. Soil-Moisture Sensor
G. IR Sensor
H. GSM Module

Software Design:

\section{A. Open Cv}

OpenCV(Open Source Computer Vision Library) is an open source computer vision and machine learning software library. The library has more than 25 languages. Computer vision allows computer to see and processed data just like human.Itinvolves analyses images to produce useful information.It is available on MAC,windows,linux.It works in $\mathrm{c}, \mathrm{C}^{++}$and python.It is easy to use and install. When you will install opencv library for python numpy will be automatically install. Numpy is general purpose array package library.

\section{B. SMTP Protocol}

SMTP(Simple Mail Transfer Protocol) is absolute ASCII Protocol which is hub as client-server model.After setup the TCP relation, the transmit machine, operating as the client, waits for taking machine operating as the server begins by transmitting a line of text delivery its individuality and convincing whether or not it is fitted to receive mail. If it is not client promulgate the connection then tries it again later.

If server is fain to accept email, the client declares whom the email is coming from to final destination. The server gives the client the go ahead message then the client sends message and server concedes it. Python gives 'smtlib' module which mark off an SMTP client session object that can be used to send mails to any internet connection with an SMTP or ESMTP listener daemon.

An SMTP object has an exemplar method called sendmail, which works on mailing a message, Fortransferring the mail you can utilize smtobj to connect to the SMTP server on local machine then utilize the sendmail method forth the message the form of band final address as arbitrary.

\section{Python}

Python is fastest growing languages in term of developers, libraries and application. That it can be used. It could be machine learning, Artificial Intelligence(AI),web development anything that you can think of.It is general purpose programming language high level which is interpreted language with easy syntax and semantics. It is created by Guido Van Rossum. He wanted to language easy to locate and easy to handle. As it was slow as compare to other languages it was not that much popular.Now with the riser of Machine Learning, Artificial Intelligence python has come out into the spotlight because it makes work much more productive and much moreeasy. It also support process oriented programming and also objective support. It is open source language. Python is a true object oriented language amd is available on a wide variety of platform. There's even a python interpreter written entirely in Java, further enhancing python's position as an excellent solution for internet based problems.

Python Statement do not need with a special character the python the python interpreter knows that you are done with an individual statement by the presence of newline.

Pyhton provides you with a certain level of freedom when composing a program but there are some rules which must always be followed. One of these rules, which python uses indentation (that is the amount of white space before the statement itself) to indicate the presence of loops, instead of using delimiters like curly braces or keywords (like 'begin' and 'end') as in many language.

Most python programmer prefer to use an editor like emacs which automatically provides consistent indetation.

\section{Database}

A database is a drive of storing data. Database are specifically designed to be very fruitful in storing and recover data. The most ordinary way of structuring data in a database is relational database system. We are using SQL database in this system.SQL stands for structural Query Language developed for accessing and modifying relational databases.

SQL is free and very widely used. It is case insensitive although field names are case sensitive.At the SQL prompt we can write in SQL instructions. Database Management system collects, stores, process and accesses data. SQL commands can be classified into three categories. These are DDL (Data Definition Language),DML(Data Manipulation Language) and TCL(Transmission Control Language) Beside SQL is commands it has various functions that performs some operation and returns a single a single value . SQL releasedunder open source and available free of cost.It has fast processing speed and easy in installation. It occupy very less space. SQL provides portability.

\section{HARDWARE USED:}

A.Power Supply: Here we used 5v/1A power supply. In this switch mode power supplies which means the output is regulated to $5 \mathrm{v}$. This power supply have a standard USB A connector for the output therefore we can power Raspberry pi through a USB cable. It regulates stable voltage. It can adapt power like mobile charger therefore it is pocket friendly.

B.Memory Card: We have used 32 GB microSD card which will be able to write at 20 mbps. where we can store data which will work for operating system.

C.Camera: We used 16mp USB camera for capturing image of crop. It has high resolution webcam which have built-in microphone. This webcam featured with night vision mode. It has manual switch for LED.It has cmos image sensor with $640 \times 480$ video resolution. It has a clip-on design for easy mounting.

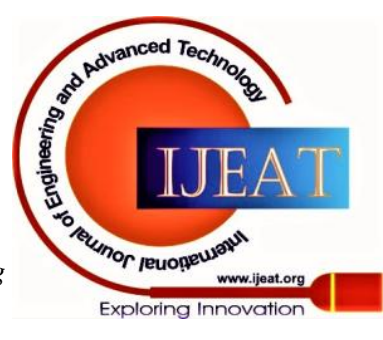


D.Enclosure: It is used to storedall hardware we use $11.81 \times 7.87 \times 3.54$ " dimension for storage.

E.Connecting wire: It gives a medium to an electrical current so that current can travel from one source on a circuit to another source.

Connecting wire utilizes to extend the firing line in an electric blastic circuit. It has insulation surrounding the metallic core.

F.Soil moisture sensor: Moisture sensor measure the water level in soil. It has two long conductor, signal gives analog value proportional to amount of moisture in the soil.Two electrode inserted into soil. The soil is more conductive it means that it has more moisture. The voltage value is potential drop at the electrode. This sensor can be used to test the moisture of soil, when the soil is having water shortage, the module output is at high level, else the output is at low level. By using this sensor one can automatically water the flower plant, or any other plants requiring automatic watering technique. Module triple output mode, digital output is simple, analog output more accurate, serial output with exact readings

G. IR Sensor: We used IR Sensor for capturing images through webcam. The main function of IR sensor is to detect obstacle. whenever leaf comes in front of IR sensor it shows high light and capture light immediately.

H. GSM Module: It works with a GSM wireless network.we are using GSM 900A .

\section{PROPOSED METHOD}

As we all know farmer faces lot of problems related to leaf disease. Some farmers didn't understand treatment on such leaf diseases. our project based on this issue.Farmers faced lot of problem regarding with leaf disease sometimes even they didn't understand exactly which disease occurred on a plant. Also, in remote areas many people didn't get pesticides in appropriate rate. With the help of this system we can resolve this problem.

In our system we have given solution to farmer which fertiliser used for plant to cure the disease on a leaf disease via Email or Short Message Service(SMS).when camera is captured the image, Indicator gives red beam of light while processing is done it gives green beam of light. Moisture sensor can be used to detect the exact level of moisture and such disease associated or occurred due to the high level of moisture content can be detected and cured.

At first ,farmer captures the image then this input goes to raspberry pi(os).Image processing is done with the help of python programming then algorithm detects and identifies the disease. For this purpose we have stored four disease images of leaf diseases. Furthermore, this information sent to fertilizer's company through email. They give effective fertiliser information to farmers then this information sent to the farmers by SMS service.This Project is not only useful for farmers but also for the fertilizer companies to find out solution. In this way appropriate information of fertilizer sent to the farmer via SMS service.For easy to capture image we had connected IR sensor to the system.

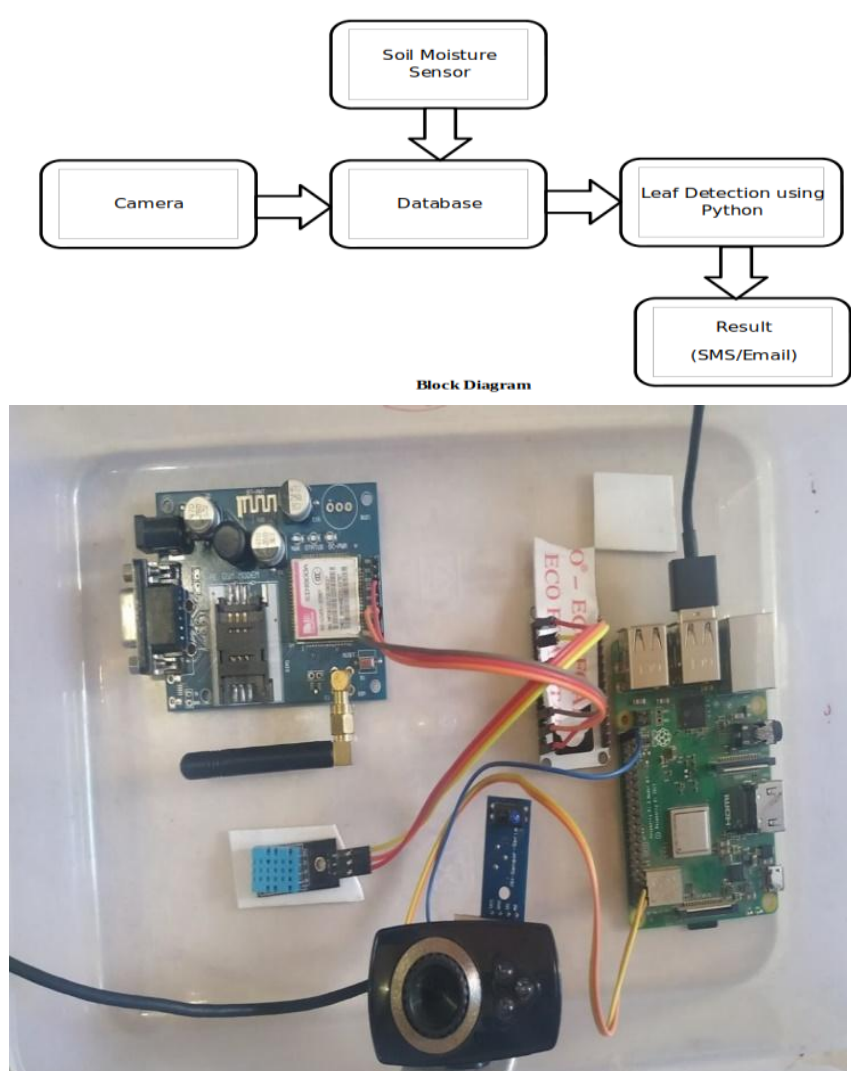

Final setup of system

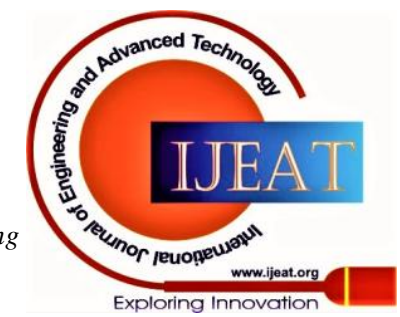




\section{Smart Solution for Agriculture Leaf Disease}

\section{Flow Chart}

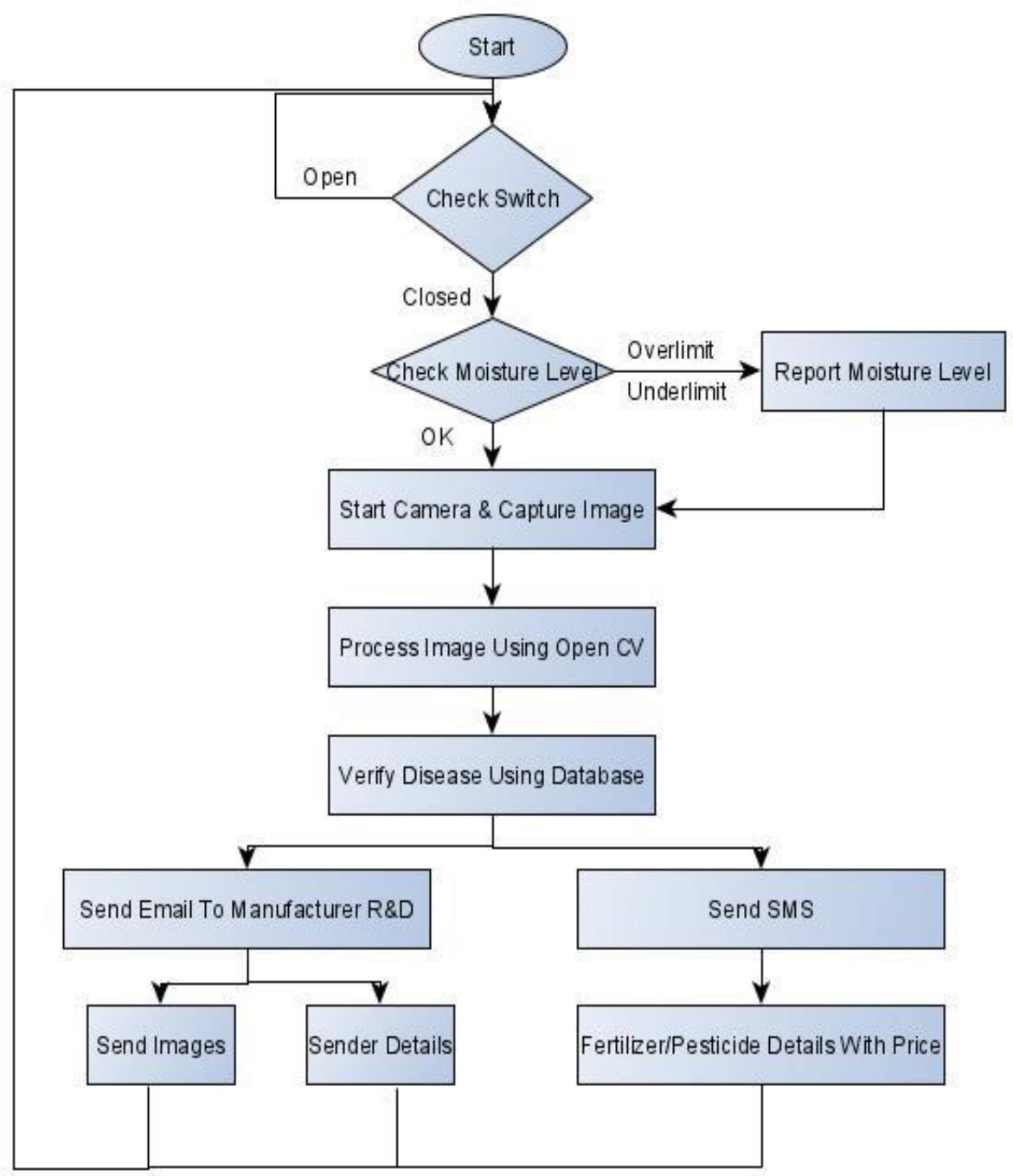

\section{RESULT}

For result purpose we have taken cotton plant leaf. The result images are as follows:

\begin{tabular}{|c|c|c|}
\hline Image Taken & Disease on cotton plant & Experimental Result \\
\hline & & Pifhon 353 Shell \\
\hline & & File Eodt Shell Debug Qpotions Window Help \\
\hline & Healthy Leaf & $\begin{array}{l}\text { Model loaded successfully, } \\
\text { Status: Healthy, } \\
\text { »» }\end{array}$ \\
\hline
\end{tabular}

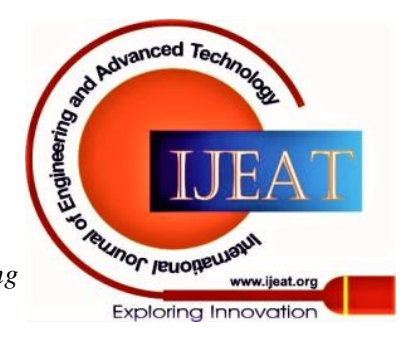




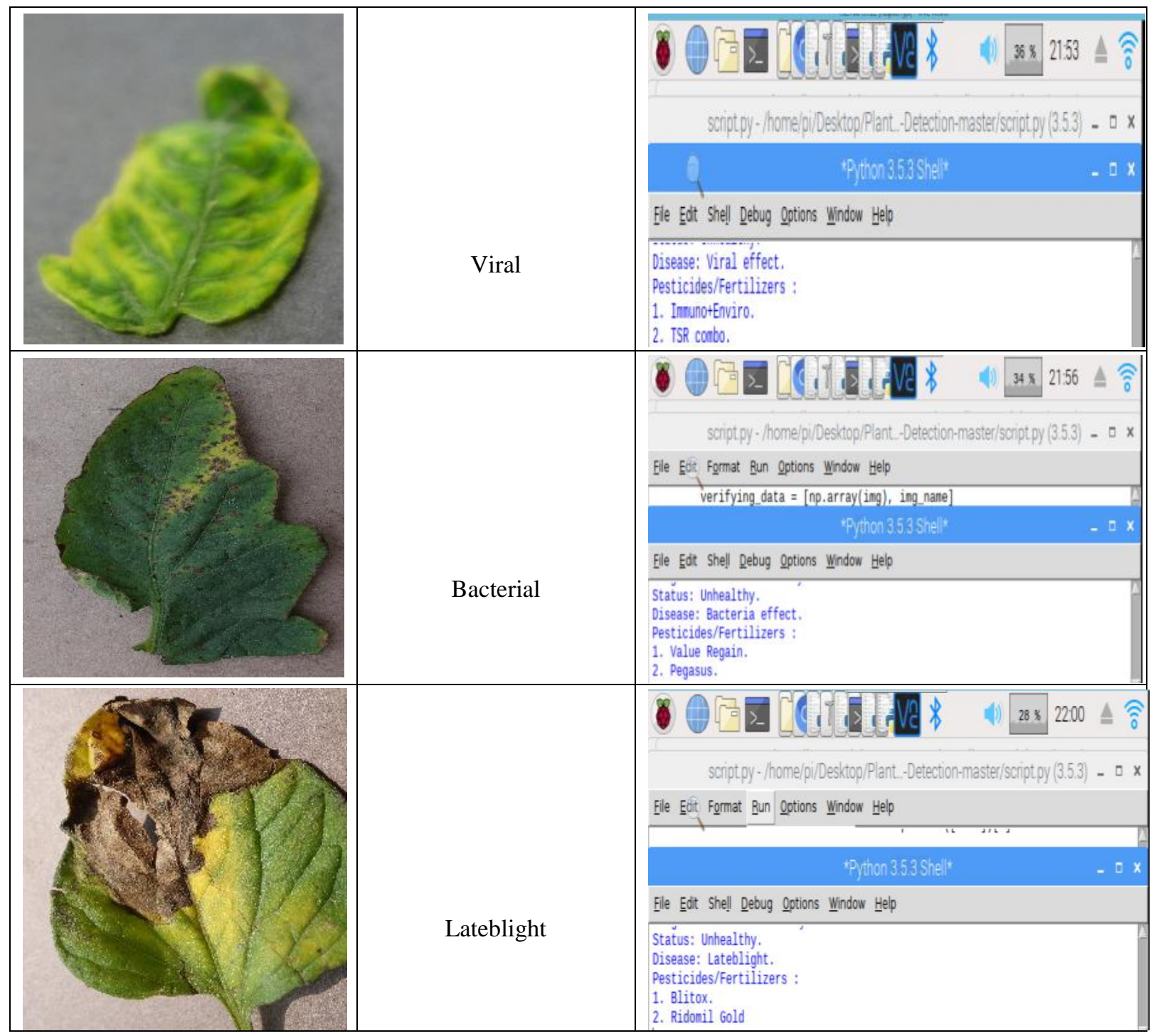

\section{CONCLUSION}

All Implementation and experiments proved that this project is a solution to the leaf detection treatment to leaf diseases.Thus, Our paper proposed cost effective system for farmers who didn't know exact name of fertilizer/pesticides. We are trying to give appropriate and good quality pesticides also accurate leaf disease detection.one more parameter we have added that is the moisture level of soil. This is also considerable forcrop development. For experimental purpose we stored pesticide and leaf disease data of cotton plant.This is time effective system also it will be available in reasonable manner so that farmer can afford it easily.This system is easy to operate. The system is beneficial for farmers of any region. The system will also reduce efforts of farmers and it is very user friendly product. The system is compact in size.

\section{FUTURE SCOPE}

Our project can be modified by using a temperature sensor, humidity sensor, DHT-11 sensor so that farmer get more benefit. A water meter can be added to evaluate the amount of water utilize for irrigation purpose.

\section{REFERENCE}

1. Precision Agriculture by TenryBrase

2. Designing Urban Agriculture by April Philips

3. Big data in Agriculture Dr. Krishnan Umachandran

4. http://en.wikipedia .org/wiki/precision agriculture

5. The International Bank for Reconstruction and Development / The World Bank "ICT in agriculture - Connecting Smallholders to Knowledge,Networks, and Institutions" e-sourcebook, 2011.

6. Smart agriculture system using IOT technology, International Journal of Recent Technology and Engineering ISSN;2277-3878,volume-7 Issue-5 January 2019

7. IOT based drone for improvement of crop quality n agricultural field by Arnab Kumar Saha

8. IOT based monitoring system in smart agriculture by Pratibha, 2017 International conference on recent advances in electronics and communication technology

9. A model for smart agriculture using IOT by Prof. K.A.Patil, 2016 International conference on global trends in signal processing.

10. IOT based agriculture monitoring and smart irrigation system using Raspberry pi by T. Vineela International Researchjournal of engineering and technology volume 5, Issue $1^{\text {st }}$ jan 2018

11. Agricultural Plant Leaf Disease Detection Using Image Processing by Prof. Sanjay B. Dhaygude, International Journal of advanced research in electrical electronics and instrumentation engineering vol.2
Published By:

Blue Eyes Intelligence Engineering and Sciences Publication

(C) Copyright: All rights reserved.

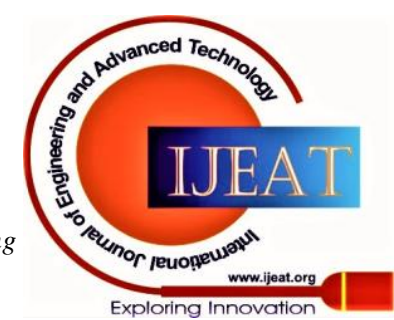




\section{Smart Solution for Agriculture Leaf Disease}

\section{AUTHORS PROFILE}

Ms. Rajlaxmi P. Chavan, MTech Student, Electronics and Telecommunications Engineering CSMSS Chh. Sahu College of Engineering Aurangabad.

Prof. A.M.Rawate, Head of Department Electronics and Telecommunication Engineering CSMSS Chh. Sahu College of Engineering Aurangabad.

Prof. A.S. Paymode, Assistant Professor, Electronics and Telecommunication Engineering CSMSS ChhSahu College of Engineering Aurangabad.

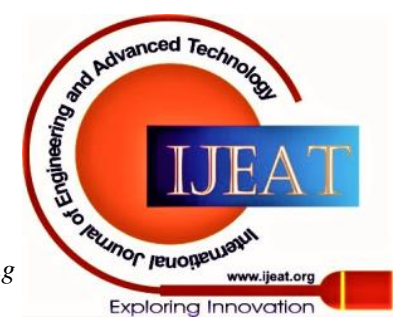

